\title{
Full-color persistent luminescence tuning: A marriage of perovskite quantum dots and lanthanide ions
}

\author{
Xiaoyong Huang
}

In modern society, the developments of luminescent materials capable of emitting various colored lights are critically important for lighting and display systems [1-3]. As a typical kind of luminescent materials, the persistent luminescence phosphors have been widely investigated as night-light vision materials due to their many significant applications such as emergency route signage and security signals, bio-labels, photocatalysts, anti-counterfeiting, optical sensors, and optical data storage [4-6]. However, realizing multicolored persistent luminescence phosphors has been a long-standing challenge, thus posing a serious problem in their practical applications $[7,8]$. In a recent publication in Angewandte Chemie International Edition, Prof. Xueyuan Chen and co-workers demonstrated a simple but effective approach to fine-tuning the persistent luminescence colors, based upon the composites of lanthanide ions doped $\mathrm{CaAl}_{2} \mathrm{O}_{4}: \mathrm{Eu}^{2+}, \mathrm{Nd}^{3+}(\mathrm{CAO})$ blue persistent phosphors with all-inorganic $\mathrm{CsPbX}_{3}(\mathrm{X}=\mathrm{Cl}, \mathrm{Br}$, and I) perovskite quantum dots (PeQDs) [9]. With the help of the radiative energy transfer from CAO blue persistent phosphors to $\mathrm{CsPbX}_{3} \mathrm{PeQDs}$, the persistent luminescence colors can be precisely controlled through tailoring of the PeQDs bandgap. The approach allowed access to full-spectrum persistent luminescence with wavelengths covering the entire visible spectral range by single-wavelength excitation, thus offering new opportunities for persistent luminescence materials in many important emerging applications.

In their experiment, Chen and co-workers used a facile spin-coating method to prepare the CAO/PeQDs/polydimethylsiloxane (PDMS) composite films (Fig. 1a and b). The authors found that the CAO blue persistent luminescence phosphors as energy storage materials can sensitize the $\mathrm{CsPbX}_{3}$ PeQDs (as light conversion materials), because the persistent luminescence of CAO matched well with the absorption of $\mathrm{CsPbX}_{3} \mathrm{PeQDs}$. Indeed, these CAO/PeQDs/PDMS composite films showed bright persistent luminescence after irradiation with a $365 \mathrm{~nm}$ UV lamp for $5 \mathrm{~min}$ (Fig. 1c), and their emission colors could be tuned from blue to green and deep red by varying the halide composition of the PeQDs from $\mathrm{Cl}$ to $\mathrm{Br}$ and I (Fig. 1d). Impressively, the persistent luminescence intensity of the films remained very high after $8 \mathrm{~h}$ of decay (Fig. 1e) and the persistent luminescence can still be clearly seen by naked eye even after $3 \mathrm{~h}$ of decay. Moreover, Chen and co-workers revealed that the obtained persistent luminescence should be attributed to the exciton emissions of $\mathrm{CsPbX}_{3}$ PeQDs through radiative energy transfer from CAO. Obviously, the large absorption coefficient and high photoluminescence efficiency of $\mathrm{CsPbX}_{3} \mathrm{PeQDs}_{\mathrm{P}}$ facilitated the efficient energy transfer from CAO to PeQDs. Due to the narrow spectral bandwidth of PeQDs, highly saturated RGB colors of persistent luminescence were achieved with a wide color gamut as high as $130 \%$ NTSC (Fig. 1f). Notably, the authors further demonstrated that the proposed light conversion strategy for persistent luminescence tuning can be applied to other energy storage and light conversion materials, and finally white-emitting persistent emission was realized.

In summary, this work presented by Chen and colleagues made a big breakthrough in persistent luminescence multicolor fine-tuning, thereby providing new chances for persistent luminescence phosphors in various novel and important photonic application fields. The light conversion strategy for color tuning of persistent luminescence proposed by Chen and co-workers could be easily extended to other luminescent material systems. Importantly, these findings will inspire more research works on developing new light-emitting functional composite materials based on the combination of lanthanide ions and all-inorganic PeQDs, and discovery of

College of Physics and Optoelectronics, Taiyuan University of Technology, Taiyuan 030024, China

Email: huangxy04@126.com 

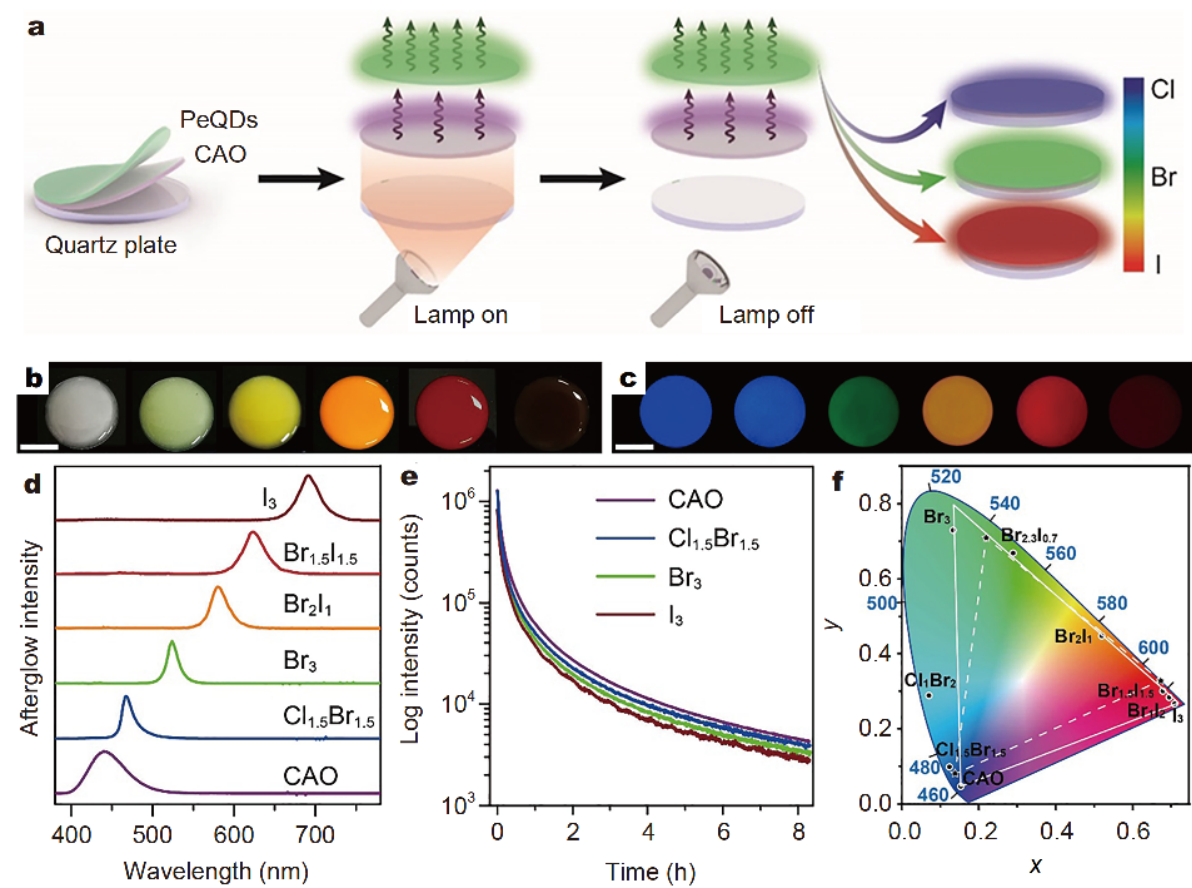

Figure 1 (a) Illustration for full-color persistent luminescence tuning in CAO/PeQDs composite films. (b) Digital photographs of CAO/PeQDs/ PDMS films with varying halide compositions of $\mathrm{CsPbX}_{3}$ PeQDs under daylight. (c) Persistent luminescence images of the films in (b) recorded at a delay time of $10 \mathrm{~min}$ after $5 \mathrm{~min}$ of illumination with a $365 \mathrm{~nm}$ UV lamp. (d) Normalized persistent luminescence spectra of CAO/PeQDs/PDMS films with various PeQDs compositions. (e) Persistent luminescence decay curves of CAO/PeQDs/PDMS films with different PeQDs compositions. (f) CIE chromaticity diagram of the persistent luminescence colors from CAO/PeQDs/PDMS films. Adapted by permission from Ref. [9], Copyright 2019, Wiley.

new luminescence phenomena and novel application areas might be expected in the near future.

Received 30 August 2019; accepted 5 September 2019; published online 27 September 2019

1 Huang X. Red phosphor converts white LEDs. Nat Photon, 2014, 8: 748-749

2 Huang X. New red phosphors enable white LEDs to show both high luminous efficacy and color rendering index. Sci Bull, 2019, 64: 879-880

3 Sun L, Devakumar B, Liang J, et al. Highly efficient $\mathrm{Ce}^{3+} \rightarrow \mathrm{Tb}^{3+}$ energy transfer induced bright narrowband green emissions from garnet-type $\mathrm{Ca}_{2} \mathrm{YZr}_{2}\left(\mathrm{AlO}_{4}\right)_{3}: \mathrm{Ce}^{3+}, \mathrm{Tb}^{3+}$ phosphors for white LEDs with high color rendering index. J Mater Chem C, 2019, 7: 1047110480

4 Zhuang Y, Wang L, Lv Y, et al. Optical data storage and multicolor emission readout on flexible films using deep-trap persistent luminescence materials. Adv Funct Mater, 2018, 28: 1705769

5 Li Y, Gecevicius M, Qiu J. Long persistent phosphors-from fundamentals to applications. Chem Soc Rev, 2016, 45: 2090-2136

$6 \mathrm{Xu} \mathrm{J}$, Tanabe S. Persistent luminescence instead of phosphorescence: History, mechanism, and perspective. J Lumin, 2019, 205: 581-620

7 Chen W, Wang Y, Zeng W, et al. Long persistent composite phosphor $\mathrm{CaAl}_{2} \mathrm{O}_{4}: \mathrm{Eu}^{2+}, \mathrm{Nd}^{3+} / \mathrm{Y}_{3} \mathrm{Al}_{5} \mathrm{O}_{12}: \mathrm{Ce}^{3+}$ : a novel strategy to tune the colors of persistent luminescence. New J Chem, 2016, 40: 485-491

8 Rodrigues LCV, Hölsä J, Lastusaari M, et al. Defect to $\mathrm{R}^{3+}$ energy transfer: Colour tuning of persistent luminescence in $\mathrm{CdSiO}_{3}$. J Mater Chem C, 2014, 2: 1612-1618

9 Gong Z, Zheng W, Gao Y, et al. Full-spectrum persistent luminescence tuning using all-inorganic perovskite quantum dots. Angew Chem Int Ed, 2019, 58: 6943-6947 